International Journal on AdHoc Networking Systems (IJANS) Vol. 7, No. 1/2/3, July 2017

\title{
THRESHOLD BASED DATA REDUCTION FOR Prolonging LifE OF Wireless SENSOR NETWORK
}

\author{
Pramod D Ganjewr ${ }^{1}$, Barani $\mathrm{S}^{2}$ and Sanjeev J. Wagh ${ }^{3}$ \\ ${ }^{1}$ Research Scholar, Sathyabama University, Chennai, Tamilnadu, India \\ Faculty, MIT Academy of Engineering, Alandi(D.), Pune, Maharashtra, India \\ ${ }^{2}$ Professor, Sathyabama University, Chennai, Tamilnadu, India \\ ${ }^{3}$ Professor, Government College of Engineering, Karad, Maharashtra, India
}

\begin{abstract}
Wireless sensor network is a set of tiny elements i.e. sensors. WSN is used in the field of Health Monitoring, Civil Construction, Military Applications and Agricultural etc., for monitoring environmental parameters. The WSN is having the challenges like less processing power, less memory, less bandwidth and battery powered. The data monitored through the sensors would be sent to the sink for data processing. The data sent from sensor node can be controlled for saving the energy, as maximum energy is consumed for transmission of data and it is not possible to replace the batteries frequently. In this work threshold based and adaptive threshold based data reduction techniques with energy efficient shortest path are used for minimizing the energy of sensor node and the network. Adaptive approach saves energy and reduce data by $30 \%$ to $40 \%$ as compared to threshold based approach.
\end{abstract}

\section{KEYWORDS}

Threshold, Data Reduction, Energy Conservation, Network Life, Wireless Sensor Network etc.

\section{INTRODUCTION}

Wireless Sensor Networks consist of tiny nodes that would perform the operations of Sensing, Processing, and transmission. The maximum energy is consumed for the transmission of data to sink from sensor node. Huge amount of data gets generated in WSN, as per the sampling rate, and this data get transmitted to sink, which consumes maximum energy of the node and get drained because of the transmission of this huge data to sink. These nodes are battery equipped and having less battery capacity. The sensor nodes are deployed in such an area where human intervention is rarely possible, hence replacing the batteries is rarely possible. Prolonging the life of that sensor and indirectly prolonging the life of the network, we need to monitor the generated data and transmits only required data to sink, which would help us to save energy of the network and would prolong life of the network. The transmission will take place using radio frequency signal [1]. In this developing era sensor nodes are deployed in hostile and inaccessible site. It provides large scope of application in the fields like Disaster Monitoring, Health Monitoring, Mechanical Industry and Automobiles Industry. The WSN performs mainly three operations Sensing, Processing and transmitting using RF signals.

Wireless Sensor Network has limitations like limited communication, limited energy, less memory, limited processing power, Sensor node hardware failure, etc. As for every operation requires energy and sensors are battery equipped, hence energy consumption is the main concern in wireless sensor network. Recharging and replacing the battery is rarely possible in WSN. Thus focusing the issue of power consumption in wireless sensor network. Which increases the importance of energy conservation for wireless sensor network which increase the lifetime of 
WSN. Data mining is the pre-processing strategy and data reduction belongs to that. Data reduction is reduction of the data to be transmitted from source to destination.

The rest of the paper is organized as Section 2 will focus on related work, Section 3 will elaborate the proposed work in detail, Section 4 will give formulation of energy consumption calculation, Section 5 will focus on experimental design and Section 6 will give thought process on result analysis and conclusion is given in Section 7, etc.

\section{RELATED WORK}

The issue of prolonging the life of wireless sensor network is addressed by many ways in the literature.

M. Arun Raja, V. Malathi in [1] proposed energy minimization technique for wireless sensor network using double data reduction concept. Depending on the similarity, nodes are clustered. The cluster head acquires data from various cluster heads using DPF. The DPF uses nLMS filtering technique to explore the correlation of data. The sink node receives data from cluster head. In this method temporal as well as spatial correlation would be explored, which work better than existing data reduction techniques where only temporal or spatial correlation technique is deployed. The works focuses on exploring the temporal as well as spatial correlation for refining the data prediction in the form of faster convergence and reduced data error. Inclusion of data compression at the cluster head is identified as a future work.

Tan, Liansheng, and Mou $\mathrm{Wu}$ [2] presented a hierarchical LMS prediction algorithm and protocol that can significantly speed up the convergence rate during the initial stage of the algorithm, thus reduce the amount of actual data transmitted to the sink. The HLMS predicting approach is able to predict the measured values both at the source and at the sink. Sensor nodes are subsequently required only to send those readings that deviate from the prediction by an error budget. Such data reduction strategy achieves significant power savings by reducing the amount of data transmissions among nodes. It should be noted, there is usually high bit error rate (BER), channel condition fluctuation, and handoff in a wireless link, which all cause packet loss in transmissions. Further once node or link failure (transient or permanent) occurs in a WSN, the sink will miss the actual reading when the prediction error exceeds the specified threshold. That would cause the failure of the prediction approach. Moreover, how to use the HLMS architecture into the spatial prediction of the WSN would be another subject for future research.

Eric J. Msechu, and Georgios B. Giannakis Proposed in [3], novel data reduction method for sensor node reported data or results are transmitted to the fusion center. Also, using an interval censoring method for each sensor node decides censoring measurement is based on mean square error impact on the estimator.

Mato's proposed in [4], simple linear regression for prediction based method for reducing the sensing of the sensor and reduce the data. Syed Misbahuddin proposed in [5], technique for increasing throughput through data reduction. This algorithm is deployed on a sensor as well as a sink. The technique used is, the repeated data are dropped at source and not transmitted to sink. Using this proposed data reduction algorithm brings maximum throughput with significant impact on throughput.

Amir Mohammad Roughens, Ali Movaghar, M. Yourself Naderi proposed in [6] Principal Component analysis, which is a combination of data aggregation and data prediction. In this, only intermediate nodes aggregate packets and transmits it to sink. Where as sink nodes are transmitting packets those are frequently occurring eigenvector values.

Pramod D. Ganjewar et. all in [7] proposed Threshold based data reduction in single sensor network. They have used threshold value as a difference between currently measured data and 
previously transmitted data, if the difference between them crosses the predefined threshold or how many transaction have not been sent, if the count of such transactions, if crosses the limit, then current data would be transmitted to the sink and this continued further. This concept will help us to reduce data up to a considerable level.

Pramod D Ganjewar et. all in [8] proposed Incremental Naive Bays method for prediction of the data value which is going to transmit the sink. The prediction will take place from groups of data values. Data reduction is directly proportional to the group size i.e. if group size is large, data reduction will be more. Because from $n$ items in the group only one value will be sent to the sink. It means n-1 values are dropped at the sensor node only. Which will save the energy required for transmission of n-1 data values and prolong the life of the network.

Pramod D. Ganjewar et. all in [9] proposed energy efficient Deflate technique for data compression in WSN. This is based on LZ77 and Huffman algorithm. In EEDeflate, fuzzification is used for calculating the node cost of the nodes in the path of transmission.

The literature survey motivated us to design and implement threshold based data reduction technique for multi-sensor network.

\section{Proposed Work}

The main objective of this work is to reduce the data to be transmitted from sensor to sink node, which will save the energy of the data items which are not transmitted to sink. It means if we are able to avoid data from sending to sink will give rise to an energy saving and prolong the life of the sensor, which prolong the life of the network.

In WSN maximum energy would be consumed for transmission of data from sensor to sink. We have proposed threshold based data reduction for multi sensor network. As more than one parameter need to be monitored from the environment and not all the sensors will monitor the data at a time, it starts with monitoring of one parameter only and when monitored value crosses the threshold then the only next sensor will be activated and this goes on till the last sensor. It means we have reduced the data being transmitted. Then for guaranteed transmission of data from sensor to sink, the nodes in the selected path should have sufficient energy, so before transmission of data every time in the transmission path such nodes are selected for guaranteed transmission of data.. We have proposed two data reduction techniques with strong path for data reduction.

1) Threshold based data reduction with energy efficient path (TBDREEP)

2) Adaptive Threshold based data reduction with energy efficient path (ATBDREEP)

In, system model shows in Fig. 1, where WSN is deployed, contains nodes with implementation of TBDREEP / ATBDREEP algorithm and sink nodes.

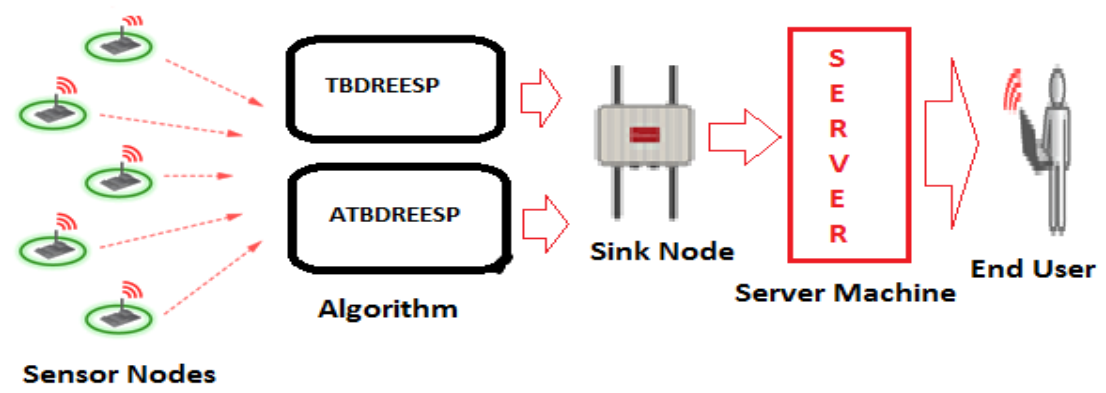

Figure 1. System Architecture 
In this model three sensor nodes are considered and that are rainfall sensor, moisture sensor and pore pressure sensor and we have implemented and compared the following algorithms

1. Threshold Based Data Reduction [TBDR]

2. TBDR using Energy Efficient Shortest Path [TBDREESP]

3. Adaptive TBDR [ATBDR].

4. Adaptive TBDR using Energy Efficient Shortest Path [ATBDREESP].

\subsection{Threshold Based Data Reduction}

In this algorithm, all the nodes sends the data to sink after crossing their threshold[10] only. When value of any sensor node crosses the threshold, that data will be sent to the sink, thus saving the energy of not transmitted transaction i.e. the data those are having value below threshold are avoided from transmission and dropped it there itself. If the sensed value $S_{v}$ is greater than threshold value $T_{v}$, then the sensed value $S_{v}$ would be sent to sink and remaining sensed values $S_{v}$, having lesser than $T_{v}$ are dropped, if number of dropped values are more, energy saving will be more, as that values are not considered for transmission.

\subsection{Threshold Based Data Reduction with Energy Efficient Shortest Path}

The concept of TBDR is used and for prolonging the life of network, energy efficient path is selected for data transmission i.e. the nodes having energy less than threshold are not considered in the path. In this algorithm, every node would make the entry of its shortest path to the sink node in its table. While considering the nodes in the shortest path, individual node should have minimum energy more than its threshold energy. If node energy $\mathrm{N}_{\mathrm{e}}$ if greater than $\mathrm{T}_{\mathrm{ne}}$, then such node will be considered in the shortest path. In between node energy $\mathrm{Ne}$ of any node goes down than its threshold energy $\mathrm{T}_{\mathrm{ne}}$, then such nodes, informs that to their source nodes that their energy is going below threshold and source nodes would update the path entry accordingly.

\subsection{Adaptive Threshold Based Data Reduction}

In the multi-sensor network, multiple sensors are deployed for monitoring many parameters, and no need to monitor all the parameters at same time[10], adaptively we would make the sensor active, as one sensor crosses its threshold and so on. At start only $S_{1}$ sensor is active and all other sensors are in ideal condition, as sensed value of $s_{1}$ crosses its threshold, then it will activate sensor $S_{2}$, then $S_{2}$ will active $S_{3}$ after crossing $S_{2}$ threshold and this will continue till $S_{n}$ th sensor.

\subsection{Adaptive Threshold Based Data Reduction with Energy Efficient Shortest Path}

In this model ATBDR is used and it follows shortest path for transferring sensor value to sink. As every node in the path should have energy more than threshold energy. If energy of any node in this shortest path goes down, it sends acknowledge to the source and source will update the shortest path before transferring the data.

These four algorithms are designed and implemented, their results are compared with in the proposed work, it is found that the algorithm which uses energy efficient shortest path for transmission of data from sensor to sink node, will performs better than their normal version.

\section{ENERgy CONSUMPTiOn CALCUlation}

Energy consumption of a sensor node and whole network would be calculated with as follows

\subsection{Energy Consumed by a Single Node}

The energy consumed by a sensor would be calculated by Eq. No. 1 . 
International Journal on AdHoc Networking Systems (IJANS) Vol. 7, No. 1/2/3, July 2017

Where

$$
\mathrm{E}_{\mathrm{sn}}=E_{p}+\sum_{i=1,2}^{T_{n}}\left(\mathrm{~B}_{\mathrm{p}} * \mathrm{E}_{\mathrm{tr}}\right)
$$

$\mathrm{E}_{\mathrm{sn}}$ - Energy consumed by a sensor.

$\mathrm{T}_{\mathrm{n}}-$ No. of transmission.

$E_{p}$ - Processing energy.

$\mathrm{B}_{\mathrm{p}}-$ Bits per sample.

$\mathrm{E}_{\mathrm{tr}}-$ Transmission Energy

\subsection{Total Energy Consumed by Network}

The total energy consumed by the network will be obtained by Eq. No. 2

Where

$$
\mathrm{E}_{\mathrm{t}}=\sum_{i=1,2}^{n} \mathrm{E}_{\mathrm{sn}}
$$

Et - Energy consumed by the network.

Esn - Energy consumed by single node.

$\mathrm{n}-$ No. of Nodes in the network.

\section{EXPERIMENTAL DESIGN}

All these algorithms i.e. NT, TBDR, ATBDR, TBDREESP, ATBDREESP are simulated in NS3. The random values are considered as a input to these algorithms. The simulation time is considered as 30 seconds and results are compared. Assumptions used for simulation are as per Table 1.

Table 1. Simulation Parameters

\begin{tabular}{|l|l|}
\hline Simulation Parameters & Description \\
\hline Topological Area & 500 x 500 Sq. mtr. \\
\hline No. of Nodes in the N/W & Up to 500 \\
\hline Network Topology & Random \\
\hline Network Type & Multi - Hopping \\
\hline Routing Protocol & AODV \\
\hline Initial Energy of the node & 10 Joule \\
\hline Packet size & 10 byte \\
\hline Simulation Time & 30 Seconds \\
\hline
\end{tabular}

\section{RESUlt ANALYSIS}

In the result analysis, all the algorithms are implemented by considering assumptions given in Table No. 1, and their results are compared. Simulation results for the network size 10, 30, 50, 70 and 90 are recorded. The track of energy consumption as per Eq. No. 1 and 2 is kept in every algorithm for simulation of network size 10, 30, 50, 70 and 90 with simulation time 30 seconds. Similarly the record of data reduction is also kept and the results are compared with existing algorithms.

\subsection{Energy Saving}

Wireless Sensor networking containing nodes 10, 30, 50, 70 and 90 are simulated and their results of energy consumption in the network are compared and their comparison is shown with the help 
of following graph. Comparison of Energy consumption, Energy Saving and \% Energy Saving by NT, TBDR, TBDREESP, ATBDR and ATBDREESP is shown in Figure 2, 3 and 4 respectively.

\subsection{Data Reduction}

Wireless Sensor networking containing nodes 10, 30, 50, 70 and 90 are simulated and their results of Data Transmission, Data Reduction and \% Data Reduction are in the network are compared. The comparison of Data Transmission, Data reduction and \% Data reduction by TBDR and TBDREESP is shown in Figure 5, 6 and 7 respectively.

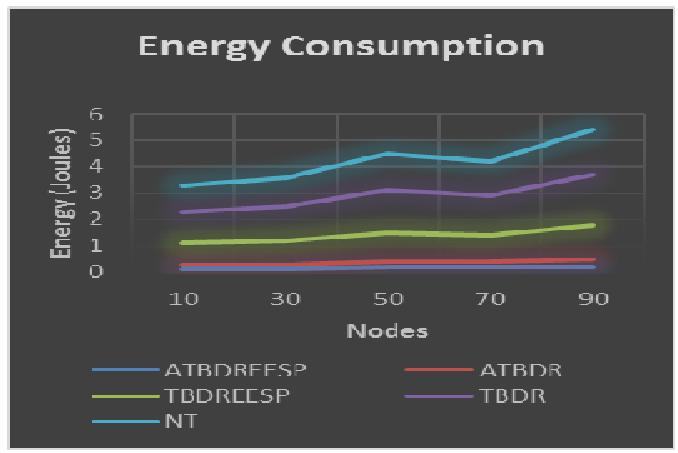

Figure 2: Energy Consumption with NT, TBDR, TBDREESP, ATBDR and ATBDREESP

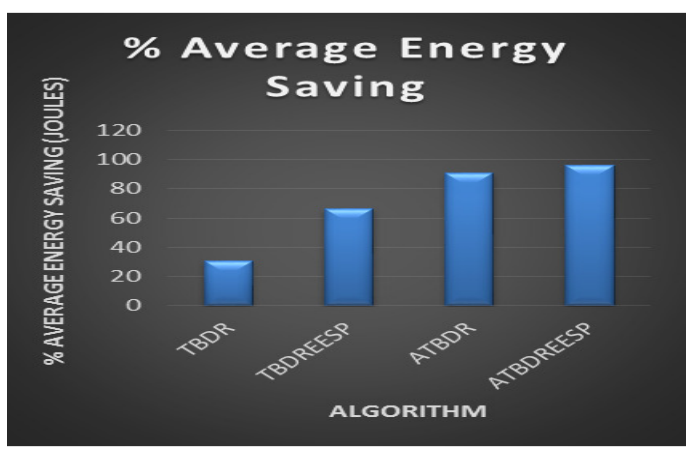

Figure 4. Energy Saving with NT, TBDR, TBDREESP, ATBDR and ATBDREESP

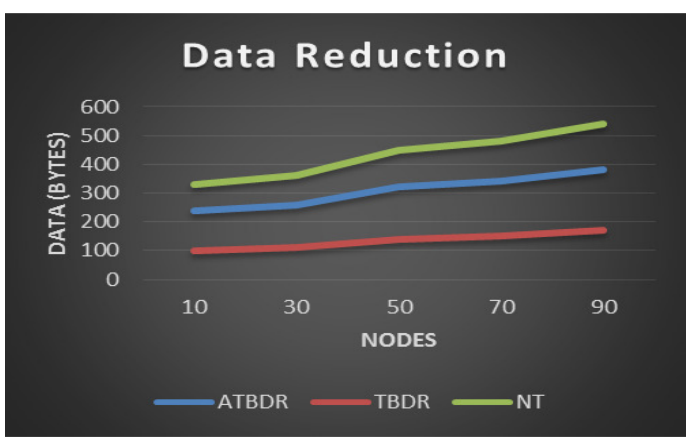

Figure 6. Comparison of Data Reduction in Bytes Using NT, TBDR and ATBDR

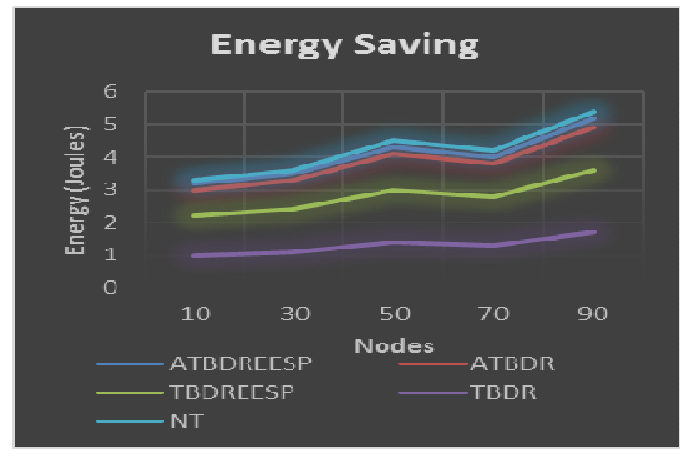

Figure 3. Energy Saving with NT, TBDR, TBDREESP, ATBDR and ATBDREESP

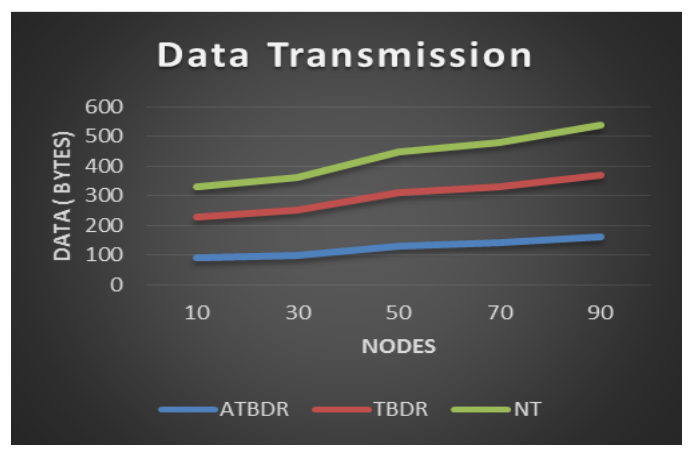

Figure 5. Comparison of Data Transmission in Bytes Using NT, TBDR and ATBDR

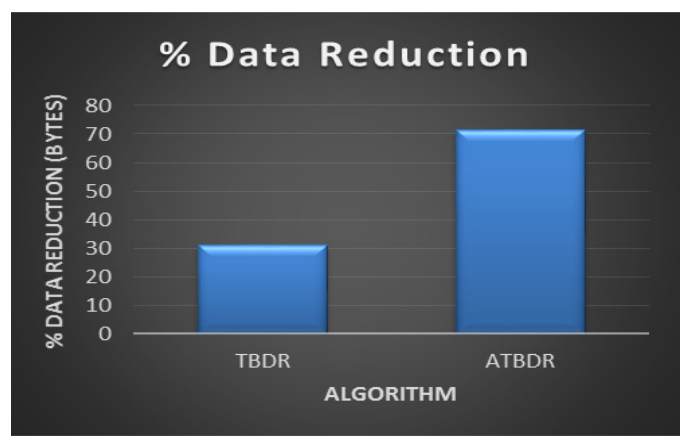

Fig. 7: Comparison of Data Reduction in Bytes Using TBDR and ATBDR 


\section{CONCLUSION}

The Energy Consumption and Data reduction of proposed algorithms TBDR, TBDREESP, ATBDR and ATBDREESP are compared with Normal Transmission first. Similarly, Energy Consumption and Data reduction by TBDR is compared with ATBDR. Also data reduction by TBDREESP and ATBDREESP are compared as TBDREESP and ATBDREESP are not contributing to Data reduction as their main focus is on saving the energy and data reduction is a secondary task.

The results for energy shaving shows that TBDREESP saves $34 \%$ to $35 \%$ of energy as compared to TBDR. Similarly ATBDREESP saves $5 \%$ to $6 \%$ of energy as compared to ATBDR. It means TBDR, TBDREESP, ATBDR, ATBDREESP prolongs the life of network as compared to normal transmission.

The energy saving is the side effect of data reduction, in the quantity of data to be sent from source to sink. The results for data reduction shows that ATBDR saves 39\% to $40 \%$ of data as compared to TBDR. This much of data reduction result in good amount of energy saving and energy saving prolongs the life of network. The results show that the type of algorithm, in which more focus is on selecting an energy efficient shortest path, would not help us to reduce data, that can be the future scope or limitations of these two proposed algorithms.

\section{ACKNOWLEDGMENT}

We thank Dr. Barani S. and Dr. Sanjeev J. Wagh for their assistance and their comments that greatly improved the manuscript. We would also like to show our gratitude to the Dr. S. S. Sonawane, Director, D. Y. Patil Knowledge City, Lohegaon Campus, Pune and Dr. B. P. Patil, Principal, Army Institute of technology, Pune for sharing their pearls of wisdom with us during the course of this research.

\section{REFERENCES}

[1] M. Arunraja, V. Malathi, E. Sakthivel, Energy Conservation in WSN through Multilevel Data Reduction Scheme, Microprocessors and Microsystems (2015), doi: http://dx.doi.org/10.1016/j.micpro 2015.05.019.

[2] Tan, Liansheng, and Mou Wu. "Data reduction in wireless sensor networks: A hierarchical LMS prediction approach." IEEE Sensors Journal 16.6 (2016): 1708-1715.

[3] Eric J. Msechu, Member, IEEE, and Georgios B. Giannakis, 400 IEEE TRANSACTIONS ON SIGNAL PROCESSING, VOL. 60, NO. 1, JANUARY 2012, Sensor-Centric Data Reduction for Estimation With WSNs via Censoring and Quantization.

[4] Matos, Brayner, A. Maia, J.E.B. Toward in-network data prediction in wireless sensor networks. In Proceedings of the ACM Symposium on Applied Computing, Sierre, Switzerland, pp. 592-596, March 2010

[5] C. Guestrin, P. Bodik, R. Thibaux R., M. Paskin and S. Madden: Distributed Regression: an Efficient Framework for Modeling Sensor Network Data". In Proceedings of the 3rd international Symposium on Information processing in Sensor Networks (IPSN '04), Berkeley, USA, April 2004.

[6] Amirmohammad Rooshenas, Hamid R. Rabiee, Ali Movaghar, M. Yousof Naderi, "Reducing The Data Transmission in Wireless Sensor Networks Using The Principal Component Analysis", sixth international conference on intelligent sensor, sensor network, information processing. 
[7] Ganjewar, Pramod D., Sanjeev J. Wagh, and S. Barani. "Threshold based data reduction technique (TBDRT) for minimization of energy consumption in WSN." Energy Systems and Applications, 2015 International Conference on IEEE, 2015.

[8] Ganjewar, Pramod D., S. Barani, and Sanjeev J. Wagh. "Data reduction using incremental Naive Bayes Prediction (INBP) in WSN." Information Processing (ICIP), 2015 International Conference on. IEEE, 2015.

[9] Ganjewar, Pramod, S. Barani, and Sanjeev J. Wagh. "Energy Efficient Deflate (EEDeflate) Compression for Energy Conservation in Wireless Sensor Network." The International Symposium on Intelligent Systems Technologies and Applications. Springer International Publishing, 2016.

[10]Mashere, Manisha P., Sunita S. Barve, and Pramod D. Ganjewar. "Energy Minimization Using Data Reduction Through Thresholding in Wireless Sensor Network." Fifth post graduate conference of computer engineering, cPGCON. 2016.

[11] Maneesha Vinodini Ramesh, Senior Member, IEEE, and Venkata Prasanna Rangan, MAY 2014. Data Reduction and Energy Sustenance in Multisensor Networks for Landslide Monitoring, IEEE SENSORS JOURNAL, vol. 14, MAY 2014.

\section{AUTHORS}

Pramod D. Ganjewar, is a Research Scholar, Sathyabama University, Chennai, Tamilnadu, India and Working as Assistant Professor, Department of Computer Engineering, MIT Academy of Engineering, Alandi(D.), Pune, Maharashtra, India. His area of research is Computer Networks, Wireless Sensor Network.

Dr. Barani S., Professor, Electrical and Communication Engineering, Sathyabama University, Chennai, Tamilnadu, India. Her area of research is Wireless Sensor Network, Computer Network and simulations.

Dr. Sanjeev J. Wagh, Professor, department of Information Technology, Government College of Engineering, Karad, Maharashtra, India. His area of research is Internet Routing, Computer Networks and Wireless sensor Network.
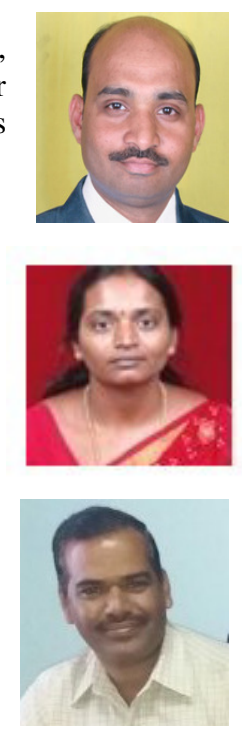